\title{
GENERALIZED DIFFUSION THEORY OF MICROMORPHICLY DEFORMABLE PARTICLE-FLUID CONTINUUM AND BUBBLE-LIQUID TWO-PHASE FLOWS
}

\author{
NGUYEN VAN DIEP \\ Institute of Mechanics, NCSR of Vietnam
}

\begin{abstract}
In the paper [1] the basic concepts and equations were derived for the micromorphicly deformable particle-fluid two-phase flows, the constitutive equations have been constructed. The flow os fluid with deformable spherical particles, when the particle only expands radially, rotates and translates, is - considered in this paper. For this case one can obtain the full motion's equations system. These equation together with the constitutive equations are sufficient to determine all of unknows, and they can be used to study the bubble-liquid two-phase flow. The problems of kinematic wave and acoustic wave propagation in the bubble-liquid two-phase flow is studied.
\end{abstract}

\section{INTRODUCTION}

The problem of multiphase flow of fluids or gase with deformable particles is attracting the great attention of scientists.

In the paper [1] a general continum theory have been developed for two-phase flows of thid with deformable particles, where the relative motion between phases and the micro-deformation of particles are taken into account.

In this paper the generalized diffusion theory, developed in [2] has been used to study the relative motion between constituents phases. The micromotion has been taken into account by generalizing the Eringen's theory $[3,4]$ of mixtures for micromorphic materials, where the micromotion of particles is considered as simple micromotion, but the micromotion of carrier fluid is kinematicly depent of particles micromotion and it is determined by extending the theory of potential motion of fuid with moving and deformable bodies [5].

The simplest case of developed theory, when the particles of spherical form only expands radially, rotates and translates is considered now.

\section{THE MOTION EQUATIONS SYSTEM}

We will consider two-phase flows of an liquid with deformable spherical particles. Suppore that during a micro-deformation process a particle can only expand radially, rotate and translate. It meand [1]

$$
\begin{aligned}
& \bar{\nu}=\nu_{0} \bar{G}+\bar{\nu}_{1} \cdot \bar{E}, \\
& \vec{I}=I_{0} \bar{G}
\end{aligned}
$$


where $\bar{\nu}$ is tensor of micro-velocity gradient, characterizing the deformaion rate of the micro-volume of particle, $\bar{\nu}_{0}$ - scalar, characterizing the radial extention rate of particle, $\bar{\nu}_{1}$ - vector of particle rotation velocity, $\bar{G}$ - unit tensor of 2 nd order, $\bar{E}$ - permutation tensor, $\bar{I}$ - tensor, characterizing the micro-inertia of particle of arbitrary form, $I_{0}$ - scalar, characterizing the micro-inertia of spherical particle.

In this case it can be shown [1] that $\Phi$ - the potential of fluid micro-motion induced in a moving spherical particle has a form:

$$
\begin{aligned}
\Phi & =\left(\bar{U}_{1}-\bar{U}_{2}\right) \cdot \bar{\Phi}_{u}+\nu_{0} \Phi_{\nu_{0}}, \\
\bar{\Phi}_{u} & =-\frac{1}{2} \frac{R^{3}}{r^{3}}, \\
\Phi_{\nu_{0}} & =-\frac{R^{3}}{r}, \quad r=|\bar{\xi}| .
\end{aligned}
$$

In the expressions (1.2) $\bar{U}_{1}$ and $\bar{U}_{2}$ are translating velocity vector of particles and liquid respectively, $\bar{\Phi}_{u}$ - potential of fluid micro-motion induced in a translation of spherical particle, $\Phi_{\nu_{0}}$ - a radially extention, $R$ - radius of particle, $\vec{\xi}$ - relative position vector of the considered fluid point with respect to the position of center mass of particle, $r$ - its absolute value.

Taking into account the results of [1] we can obtain the following expressions for the generalized induced mass tensors

$$
\begin{aligned}
\bar{M} & =M_{0} \bar{G}, \quad M_{0}=\frac{2}{3} \pi R^{3} \rho_{2} ; \\
\bar{L} & =0 ; \\
\bar{N} & =N_{0} \overline{G G}, \quad N_{0}=\frac{4}{3} \pi R^{5} \rho_{2}
\end{aligned}
$$

wher $\rho_{2}-$ mass density of liquid.

It can be shown that Io has the form

$$
I_{0}=\frac{4}{3} \pi R^{5} \rho_{1}
$$

where $\rho_{1}$ - mass density of particle.

The micro-pressure tensor $\bar{\pi}^{*}$ can be written in the form

$$
\bar{\pi}^{*}=\pi_{0}^{*} \bar{G}
$$

Then, we obtain the following motion equations system

$$
\begin{aligned}
& \frac{d^{(a)} n}{d t}+n\left(\bar{\nabla} \cdot \bar{U}_{a}\right)=-\bar{\nabla} \cdot\left(n \frac{\bar{J}^{a}}{\rho_{1}^{*}}\right) \\
& \frac{d^{(a)} \rho}{d t}+\rho\left(\bar{\nabla} \cdot \bar{U}_{a}\right)=-\bar{\nabla} \cdot\left(1-\frac{a_{1} \rho_{2}^{*}}{a_{2} \rho_{1}^{*}}\right) \bar{J}^{a} \\
& \frac{d^{(a)}}{d t}\left(\rho_{1} \varphi\right)+\rho_{1} \varphi\left(\bar{\nabla} \cdot \bar{U}_{a}\right)=-\bar{\nabla} \cdot \bar{J}^{a} \\
\rho \frac{d^{(a)} \bar{U}_{a}}{d t}= & \rho \bar{f}-\bar{\nabla} p+\bar{\nabla} \tau_{0}-\bar{\nabla} \times \bar{\tau}_{1}^{a}+\bar{\nabla} \cdot \bar{\tau}_{2}^{a}- \\
& -\frac{D^{(a)}}{D t}\left(\frac{a_{2} \rho_{1}^{*}-a_{1} \rho_{2}^{*}}{a_{2} \rho_{1}^{*}}\right)-\bar{\nabla} \cdot\left[\frac{\bar{J}^{a} \bar{J}^{a}}{\rho_{1}^{*}}\left(1+\frac{a_{1}^{2} \rho_{2}^{*}}{a_{2}^{2} \rho_{1}^{*}}\right)\right]
\end{aligned}
$$




$$
\begin{aligned}
& \frac{a_{2}^{2} \rho_{1}^{*}+a_{1}^{2} \rho_{2}^{*}}{a_{2}^{2} \rho_{1}^{*}} \frac{D^{(a)} \bar{J}^{a}}{D t}+\frac{a_{2}^{3} \rho_{1}^{*}-a_{1}^{*} \rho_{2}^{*}}{a_{2}^{3} \rho_{1}^{*}} \bar{\nabla} \cdot\left(\frac{\bar{J}^{a} \bar{J}^{a}}{\rho_{1}^{*}}\right)+\frac{a_{1}}{a_{2}} \bar{J}^{a} \frac{d^{(a)}}{d t}\left(\frac{a_{1} \rho_{2}^{*}}{a_{2} \rho_{1}^{*}}\right)- \\
& -\frac{a_{1}}{a_{2}} \frac{\bar{J}^{a}}{\rho_{1}^{*}}\left(\bar{J}^{a} \cdot \bar{\nabla}\right)\left(\frac{a_{1}^{2} \rho_{2}^{*}}{a_{2}^{2} \rho_{2}^{*}}\right)+n\left(1+\frac{a_{1}}{a_{2}}\right) M_{0}\left\{\frac{d^{(a)}}{d t}\left[\left(1+\frac{a_{1}}{a_{2}}\right) \frac{\bar{J}^{a}}{\rho_{1}^{*}}\right]+\right. \\
& \left.+\left(\frac{\bar{J}^{a}}{\rho_{1}^{*}} \cdot \bar{\nabla}\right)\left[\left(1+\frac{a_{1}}{a_{2}}\right) \frac{\bar{J}^{a}}{\rho_{1}^{*}}\right]\right\}+\frac{n}{2}\left[\frac{d^{(a)} M_{0}}{d t}+\left(\frac{\bar{J}^{a}}{\rho_{1}^{*}} \cdot \bar{\nabla}\right) M_{0}\right] \bar{J}^{a}= \\
& =\rho_{1}^{*}\left(\bar{f}_{1}-\frac{a_{1} \rho_{2}^{*}}{a_{2} \rho_{1}^{*}} \bar{f}_{2}\right)-\rho_{1}^{*}\left(1-\frac{a_{1} \rho_{2}^{*}}{a_{2} \rho_{1}^{*}}\right) \frac{d^{(a)} \bar{U}_{a}}{d t}- \\
& -\rho_{1}^{*}\left(1+\frac{a_{1}}{a_{2}}\right)\left(\bar{\nabla} \mu_{1}\right) p_{p, \pi_{0}, T}-\varphi\left[1-\frac{a_{1}(1-\varphi)}{a_{2} \varphi}\right] \bar{\nabla} p- \\
& -\rho_{1}^{*}\left(1-\frac{\rho}{\rho_{1}^{*}}-\frac{a_{1} \rho_{2}^{*}}{a_{2} \rho_{1}^{*}}\right)\left(\bar{\nabla} I_{0}\right) \pi_{0}-\rho_{1}^{*} \bar{F}_{a}^{*} ; \\
& n N_{0}\left(\frac{d^{(a)} \nu_{0}}{d t}+\frac{5}{2} \nu_{0}\right)+n I_{0}\left(\frac{d^{(a)} \nu_{0}}{d t}+\nu_{0}^{2}\right)+n\left(N_{0}+I_{0}\right)\left(\frac{\bar{J}^{a}}{\rho_{1}^{*}} \cdot \bar{\nabla}\right) \nu_{0}= \\
& \quad=\rho_{1}^{*} \ell_{0}+\pi_{0}^{*}-\chi_{0}+\bar{\nabla} \cdot \bar{\lambda}_{12}, \\
& n I_{0}\left(\frac{d^{(a)} \bar{\nu}_{1}}{d t}+\nu_{0} \bar{\nu}_{1}\right)+n I_{0}\left(\frac{\bar{J}^{a}}{\rho_{1}^{*}} \cdot \bar{\nabla}_{)} \bar{\nu}_{1}=\right. \\
& \quad=\rho_{1}^{*} \bar{\ell}_{1}+\bar{\tau}_{1}^{a}+\bar{\nabla} \lambda_{0}-\frac{1}{2} \bar{\nabla} \times \bar{\lambda}_{11}+\bar{\nabla} \cdot \bar{\lambda}_{21} ;
\end{aligned}
$$

In the equations system (1.6), the first is particle number conservation equation, the 2nd mixture mass conservation, 3th - particle mass conservation, 4th - mixture momentum balance, 5 th - particle generalized diffusion, 6th - particle volume cìange, 7th - particle rotation one.

In obtaining equations (1.6) we suppose that

$$
\begin{aligned}
& \rho_{2}=\text { const } \\
& \frac{4}{3} \pi R^{3} \rho_{1}=\text { const; }
\end{aligned}
$$

Moreover we have the following relationships

$$
\begin{aligned}
& \nu_{0}=\frac{1}{R}\left[\frac{d^{(a)} R}{d t}+\left(\frac{\bar{J}^{a}}{\rho_{1}^{*}} \cdot \vec{\nabla}\right) R\right] \\
& \varphi=\frac{4}{3} \pi R^{3} n
\end{aligned}
$$

In (1.6) - (1.8) $n$ is number of particles in a unit volume of the flow; $\bar{U}_{a}$ - certain characteristic mean velocity, expressed linearly in terms of liquid and particles velocities by some system of normalized weighing factors $a_{k} ; \vec{J}^{a}$ - generalized diffusion flux of particles; $\rho_{1}^{*}$ and $\rho_{2}^{*}$ - fictive mass densities of particle and liquid respectively; $\rho$ - mean mass density of mixture; $\varphi$ - volume concentration of particles; $\bar{f}$ - density of the external body force acting on mixture; $\bar{f}_{1}$ and $\bar{f}_{2}$ - density of the external body force acting on particles and liquid respectively; $\mu_{1}$ - generalized chemical potential; $p$ - thermodynamical pressure; $\bar{\tau}_{1}^{3}, \bar{\tau}_{2}^{a}, \bar{F}-a^{*}, \chi_{0}, \lambda_{0}, \bar{\lambda}_{11}, \bar{\lambda}_{12}, \bar{\lambda}_{21}$ - general. ized thermodynamical forces; $d^{\{a\}} / d t$ - full time derivative determining the change over time with respect to a coordinate system moving at the characteristic velocity $\bar{U}_{a}$; the operator $D^{(a)} / D t$. time derivative determining the change over time with respect to a generalized Lagrangian coordinate system moving and deforming at velocity $\bar{U}_{a}[1]$; the operator () - scalar product and $(x)$ - vector product between two closest indices of tensor quantities. 
Together with the constitutive equations [1], the equations system (1.6) - (1.8) can be considered as the equations of motion of the fluid-deformable spherical particle two-phase media. In particular they can be used for predicting the bubble-liquid two-phase flows. This equations system will be analyzed in the next chapter.

\section{SIMPLE CASED OF BUBBLE-LIQUID TWO-PHASE FLOWS}

a) Case 1:

Consider the case when there is no relative motion between phases, the spherical particle only expands radially, the liquid mass density and the total mass of a particle are constant, the motion is isothermal, and the dissipative terms can be neglected. Then, it can be shown that the equations of motion have the form

$$
\begin{aligned}
& \frac{d n}{d t}+n \bar{\nabla} \cdot \bar{U}=0 ; \\
& \frac{d}{d t}\left(\rho_{1} \varphi\right)+\rho_{1} \varphi(\bar{\nabla} \cdot \bar{U})=0 \\
& \varphi=\frac{4}{3} \pi R^{3} n ; \quad \rho_{2}=\text { const; } \\
& \frac{4}{3} \pi R^{3} \rho_{1}=\text { const; } \quad \frac{d}{d t}(\ldots)=\frac{\partial}{\partial t}(\ldots)+(\bar{U} \cdot \bar{\nabla})(\ldots) \\
& \frac{d \bar{U}}{d t}=\rho \bar{f}-\bar{\nabla} p ; \quad \\
& \frac{4}{3} \pi R^{3} n \rho_{2}\left[R \frac{d^{2} R}{d t^{2}}+\frac{3}{2}\left(\frac{d R}{d t}\right)^{2}\right]+\frac{4}{3} \pi R^{3} n \rho_{1} \frac{R d^{2} R}{d t^{2}}=\pi_{0}^{*}
\end{aligned}
$$

In (2.1) the last equation determines the change of the paricle radius $R$. It is necessary to note that the micropressure $\pi_{0}^{*}$ has to be determined by giving a concrete form of the internal energy of two-phase flow [1]. But, here in comparing with the Rayleigh's equation [6] we can set

$$
\pi_{0}^{*}=\frac{4}{3} \pi R^{3} n=\left(p_{1}-p-\frac{2 \sigma}{R}\right)
$$

where $p_{1}$ is the gas (or vapor) pressure inside a particle, $\sigma$ - the surface tension.

In addition, it is necessary to consider the equation of state for the gas (or vapor) inside the particle. As usual [6], we may assume the following state equation

$$
\frac{p_{1}}{\rho_{1}}=\mathrm{const}
$$

Equations (2.1) - (2.3) are sufficient to determine all the unknows.

b. Case 2:

When there is no particle rotation, the mean - volume velocity of the two-phase. Flow is used, the volume concentration of particles is small, the mass density of gas (or vapor) can be neglected with respect to the liquid mass density, the dissipative terms in the momentum balance equation and the particle radial expansion equation can be neglected, then the equations of motion will have the following form

$$
\begin{aligned}
& \frac{d^{(\nu)}}{d t}\left(\rho_{1} \varphi\right)+\rho_{1} \varphi \bar{\nabla} \cdot \bar{U}_{\nu}=-\bar{\nabla} \cdot \bar{J}^{\nu} \cdot \\
& \bar{\nabla} \cdot \bar{U}_{\nu}=\frac{1}{1-\varphi}\left(\frac{d^{(\nu)} \varphi}{d t}+\bar{\nabla} \cdot \frac{\bar{J}^{\nu}}{\rho_{I}}\right)
\end{aligned}
$$




$$
\begin{aligned}
& (1-\rho) \rho_{2} \frac{d^{(\nu)} \bar{U}_{\nu}}{d t}=(1-\varphi) \rho_{2} \bar{g}-\bar{\nabla} p+\rho_{2} \frac{R^{(\nu)}}{D t}\left(\frac{\bar{J}^{\nu}}{\rho_{1}}\right) ; \\
& \frac{d^{(\nu)} \bar{J}^{\nu}}{d t}+\left(\frac{\bar{J}^{\nu}}{\rho_{1} \varphi} \cdot \bar{\nabla}\right) \bar{J}^{\nu}=-2 \varphi \rho_{1}\left(\bar{g}-\frac{d^{(\nu)} \bar{U}_{\nu}}{d t}\right)-\frac{2 \varphi \rho_{1}^{2}}{\rho_{2}}\left(\bar{\nabla} \mu_{1}\right)_{p, \pi_{0}, T}-\frac{2 \varphi \rho_{1}^{2}}{\rho_{2}} \alpha \bar{J}^{\nu} \\
& R \ddot{R}+\frac{3}{2} \dot{R}^{2}+R\left(\frac{\bar{J}^{\nu}}{\rho_{1} \varphi} \cdot \bar{\nabla}\right) \dot{R}+\frac{3}{2}\left(\frac{\bar{J}^{\nu}}{\rho_{1} \varphi} \cdot \bar{\nabla}\right) R=\frac{1}{\rho_{2}}\left(p_{1}-p-\frac{2 \sigma}{R}\right), \\
& \varphi=\frac{4}{3} \pi R^{3} n ; \quad \frac{4}{3} \pi R^{3} \rho_{1}=\mathrm{const} ; \quad \frac{t_{1}}{\rho_{1}}=\mathrm{const}
\end{aligned}
$$

where

$$
\begin{aligned}
& \bar{U}_{\nu}=\varphi \bar{U}_{1}+(1-\varphi) \bar{U}_{2} ; \quad \bar{J}^{\nu}=\rho_{1} \varphi\left(\bar{U}_{1}-\bar{U}_{\nu}\right) \\
& \dot{R}=\frac{d^{(\nu)} R}{d t} ; \quad \ddot{R}=\frac{d^{(\nu)} \dot{R}}{d t} ; \quad \frac{d^{(\nu)}}{d t}(\ldots)=\frac{\partial}{\partial t}(\ldots)+\left(\bar{U}_{\nu} \cdot \bar{\nabla}\right)(\ldots) \\
& \frac{D^{(\nu)}}{D t}(\ldots)=\frac{d^{(\nu)}}{d t}(\ldots)+[(\ldots): \bar{\nabla}] \bar{U}_{\nu}+(\ldots)\left(\bar{\nabla} \cdot \bar{U}_{\nu}\right)
\end{aligned}
$$

\section{THE PROPAGATION OF KINEMATIC WAVE AND ACOUSTIC WAVE IN THE BUBBLE-LIQUID TWO-PHASE FLOWS}

To determine the velocities of a kinematic and acoustic waves propagation in the bubblenfluid two-phase flows we suppose that [4]

$$
\left(\bar{\nabla} \mu_{1}\right)_{p, \pi_{0}, T}=0, \quad p_{1}=p
$$

Then, the one-dimensional equation system (2.4) can be shown to have the following form

$$
\begin{aligned}
& \frac{\partial \varphi}{\partial t}+u \frac{\partial \varphi}{\partial x}+\frac{\varphi}{p} \frac{\partial p}{\partial t}+\frac{\varphi u}{p} \frac{\partial p}{\partial x}+\varphi \frac{\partial u}{\partial x}+\frac{1}{A p} \frac{\partial J}{\partial x}=0 \\
& \frac{\partial \varphi}{\partial t}+u \frac{\partial \varphi}{\partial x}-\frac{J}{A p^{2}} \frac{\partial p}{\partial x}-(1-\varphi) \frac{\partial u}{\partial x}+\frac{1}{A p} \frac{\partial J}{\partial x}=0 \\
& \frac{J \rho_{2}}{A p^{2}} \frac{\partial p}{\partial t}+\left(1+\frac{\rho_{2} u J}{A p^{2}}\right) \frac{\partial p}{\partial x}+(1-\varphi) \rho_{2} \frac{\partial u}{\partial t}+\left[(1-\varphi) \rho_{2} u-\frac{2 \rho_{2} J}{A p}\right] \frac{\partial u}{\partial x}- \\
& \quad-\frac{\rho_{2}}{A p} \frac{\partial J}{\partial t}-\frac{\rho_{2} u}{A p} \frac{\partial J}{\partial x}=(1-\varphi) \rho_{2} g \\
& -2 \varphi A p \frac{\partial u}{\partial t}-2 \varphi A p u \frac{\partial u}{\partial x}+\frac{\partial J}{\partial t}+\left(u+\frac{J}{A p \varphi}\right) \frac{\partial J}{\partial x}=-\frac{2 \varphi A^{2} p^{2}}{\rho_{2}} \alpha J-2 \varphi A p g
\end{aligned}
$$

where, we put $\frac{p_{1}}{\rho_{1}}=\frac{1}{A}, A=$ const.

The characteristic equation of the system (3.1) has a form

$$
(u-\lambda)\left[-\frac{\varphi(1-3 \varphi) \rho_{2}}{p}(u-\lambda)^{3}-\frac{(1-4 \varphi) \rho_{2} J}{A p^{2}}(u-\lambda)^{2}+\left(1+\frac{\rho_{2} J^{2}}{A^{2} p^{3}}\right)(u-\lambda)+\frac{J}{A p \varphi}\right]=0
$$

From the equation (3.2) one can see that the first characteristic velocity of equations system (3.1) is equal

$$
\lambda_{1}=u
$$


When there is no relative motion between bubbles and the carrier liquid (that means $J=\sigma$ ) from the characteristic equation (3.2) we can obtain the following characteristic velocities:

$$
\lambda_{2,3}^{0}=u \pm a_{0}
$$

where

$$
a_{0}=\sqrt{\frac{p}{\varphi(1-3 \varphi) \rho_{2}}} ;
$$

It is obvious that $a_{0}$ is the velocity of acoustic wave propagation in motionless bubble-liquid two-phase media. It is coincided with the know result [6].

Taking into account of (3.3) and (3.5) the characteristic equation (3.2) can be transformed into following equation

$$
Z^{3}+\frac{1-4 \varphi}{1-3 \varphi} \varepsilon Z^{2}-\left(1+\frac{\varphi}{1-3 \varphi} \varepsilon^{2}\right) Z-\varepsilon=0
$$

where we put

$$
Z=\frac{u-\lambda}{a_{0}} ; \quad \varepsilon=\frac{J}{A p \varphi a_{0}}
$$

In general the dimensionless parameter $\varepsilon$ is small, and the equation (3.6) can be solved by the following expanding

$$
Z=Z_{0}+\varepsilon Z_{1}
$$

By this way it can be shown that the equation (3.6) has 3 following characteristic velocities

$$
\begin{aligned}
\lambda_{2,3} & =u+\frac{J}{2(1-3 \varphi) \rho_{1}} \pm a_{0}, \\
\lambda_{4} & =u-\frac{J}{(1-3 \varphi) \rho_{1}},
\end{aligned}
$$

From (3.8) the characteristic velocities $\lambda_{2,3}$ can be considered as the velocities of accustic wave propagation, $\lambda_{4}$ - the velocity of kinematic wave propagation in bubble-liquid two-phase flows.

The problem of damping or amplification of these waves during the propagation in bubbleliquid two-phase flows will be the subject of further investigations.

This Publication is completed with financial support from the National Basic Research Program in Natural Sciences.

\section{REFERENCES}

1. Nguyen Van Diep. Generalized diffusion theory of micromorphicly deformable particle-fluid continuum, Proceedings of the National Centre for Scientific Research of Vietnam, Vol. 4, No 1, (1992).

2. Nguyen Van Diep. To the generalized diffusion theory of mixtures, Int. J. Advances in Mechanics, vol. 11, No 2 (1988) (in Russian).

3. Twiss R. I., Eringen A. C. Theory of mixtures for micromorphic materials-Int J. Engng Sci, Vol. 9, 1019-1044 (1971)

4. Eringen A. C. Theory of thermo-microstrech fluids and bubbly liquids, Int. J. Engng. Sci. Vol 28, 1433-1443 (1990)

5. Batchelor G. K. An introduction to fluid dynamics, Cambridge University Press (1967).

6. Biesheuvel A., Van Wijngaarden L. Two-phase flow equations for a dilute dispersion of gase bubbles in liquid, J. Fluid-Mech, Vol. 148, 301-318 (1984) 